\title{
Germanica
}

GERMANICA $48 \mid 2011$

Max Frisch philosophe?

\section{Max Frischs politische Ethik}

Max Frisch's political ethics.

L'éthique politique de Max Frisch.

\section{Walter Lesch}

\section{OpenEdition}

\section{Journals}

Édition électronique

URL : http://journals.openedition.org/germanica/1195

DOI : 10.4000/germanica. 1195

ISSN : 2107-0784

\section{Éditeur}

Université de Lille

\section{Édition imprimée}

Date de publication : 1 juillet 2011

Pagination : $94-110$

ISBN : 9782913857278

ISSN : 0984-2632

\section{Référence électronique}

Walter Lesch, «Max Frischs politische Ethik », Germanica [En ligne], 48 | 2011, document 6, mis en ligne le 01 juin 2013, consulté le 06 octobre 2020. URL : http://journals.openedition.org/germanica/ 1195 ; DOI : https://doi.org/10.4000/germanica.1195

Ce document a été généré automatiquement le 6 octobre 2020.

(C) Tous droits réservés 


\title{
Max Frischs politische Ethik
}

\author{
Max Frisch's political ethics. \\ L'éthique politique de Max Frisch.
}

\section{Walter Lesch}

1 Dass Schriftsteller in ihrer Funktion als öffentliche Personen und als Intellektuelle eine besondere Affinität zur Politik haben, ist eine anerkannte Tatsache. Als Autoren stehen ihnen Kommunikationsmöglichkeiten offen, die sie je nach persönlichem Temperament nutzen können, um auf Debatten Einfluss zu nehmen. Ihr Urteil ist gefragt, weil sie als bekannte Identifikationsfiguren ein größeres Gewicht als andere Bürger haben, wenn es darum geht, Tendenzen zu verstärken, Missstände zu kritisieren und neue Impulse $\mathrm{zu}$ geben. Diese öffentliche Rolle muss nicht unbedingt mit einem konkreten politischen Engagement im Sinne einer parteipolitischen Präferenz gekoppelt sein. Es muss auch nicht auf einer systematisch reflektierten Position politischer Philosophie beruhen. Doch verschiedene Aspekte von all dem sind von Bedeutung, wenn es gilt, die politische Dimension eines Autors zu würdigen.

\section{Literatur, Philosophie und Politik}

2 Im Fall von Max Frisch wird deutlich, dass die Klischees von engagierter Literatur und intellektuellem Wächteramt zu kurz greifen, um die Bedeutung des Politischen in den verschiedenen Perioden seiner schriftstellerischen Tätigkeit zu erfassen. Max Frisch verweigert sich insbesondere der Zuordnung zu einer bestimmten politischen Ideologie und ist auch philosophisch nicht eindeutig zu verorten. Letzteres liegt vor allem an seiner persönlichen Distanz zu jeglicher Art von Denksystem, weil ihm der Gebrauch einer philosophischen Fachterminologie fremd ist. Die selbstkritische Einschätzung seiner Ausdrucksmöglichkeiten findet sich in einem Text, den Peter von Matt den posthum veröffentlichten Skizzen $\mathrm{zu}$ Frischs drittem Tagebuch beigefügt hat: "Allgemein beneide ich jedermann, der eine Denkschule durchlaufen hat, gleichviel welche; ob als Jesuit oder Protestant oder Marxist oder Kabbalist; auch wenn einer später seine Position ändert, er bewegt sich in einem Koordinaten-System. Wenn ich mich in Begrifflichkeit einlasse, so schwimme ich und fühle mich als Schwätzer, wobei 
es mich nicht erleichtert, wenn der andere auch ein Schwätzer ist, und das kommt vor. Ich bin auf Erfahrungen angewiesen, die mich begrifflich hilflos machen und von daher narrativ. Was sich nicht umsetzt ins Anschauliche, bleibt bei meiner Anlage immer uneigen ${ }^{{ }_{1}}$. Auf dem auf der Schreibmaschine geschriebenen und mit handschriftlichen Korrekturen versehenen Text wurde folgender Beginn vom Autor gestrichen: "Meine sogenannten Gedanken sind nie profund gewiesen. Ich bin kein philosophischer Kopf, nicht einmal ein politischer" ${ }^{\text {"2 }}$.

3 So viel Selbstzweifel und Bescheidenheit scheinen von Anfang an den Weg $\mathrm{zu}$ versperren, Max Frischs politische Ethik rekonstruieren zu wollen. Denn das wäre ja im schlimmsten Fall die Illusion einer Systematik, die der Autor nie gewollt hat und für die er sich auch nicht fähig hielt. Mit den folgenden Überlegungen soll Frischs sympathische Selbsteinschätzung respektiert werden. Sie bildet ein erfreuliches Gegenwicht zu einem arroganten Selbstbild des Schriftstellers, der sich automatisch mit der Rolle des Intellektuellen und Philosophen identifiziert, obwohl diese Funktionen nicht in einer Person vereint sein müssen, um ein guter Schriftsteller zu sein. Das von Frisch erwähnte "Koordinaten-System" der Begrifflichkeit kann nämlich zur Falle werden, wenn man komplexe Begriffe mit viel Imponiergehabe benutzt und den Leser durch einen unverständlichen Stil oder einen Jargon zu beeindrucken versucht. Zu Frischs Zeiten war das wohl der Preis, der zu zahlen war, um den Zutritt zu der jeglicher Alltagsrealität entrückten Welt mancher philosophischer Systeme zu erhalten. Sollte der Obskurantismus einer skurrilen Sprache die Bedingung dafür sein, die eigenen Gedanken für "profund" zu halten, so wird man getrost auf den vermeintlichen Tiefsinn der Philosophie verzichten können, zumal im Bereich der politischen Philosophie längst ein verständlicherer Stil Einzug gehalten hat $^{3}$. Im Typoskript des zitierten Textes hat Frisch ebenfalls folgenden Satz durchgestrichen: "Vor Hegel bin ich verloren; da hat es gar keinen Sinn, dass ich weiterlese“ ${ }^{\text {" }}$. Käme der Autor heute in Berührung mit der praktischen Philosophie, so fände er hoffentlich genügend Texte, die mit dem Pathos der klassischen Tiefsinn-Philosophen nichts mehr gemein haben und die im Gegenteil Verständlichkeit und Anschaulichkeit als Gütemerkmale philosophischer Argumentation anerkennen. In der Selbstanalyse Max Frischs spiegelt sich die Charakterisierung eines unverwechselbaren Stils, der selbstkritisch als begriffliche Hilflosigkeit bezeichnet werden kann, positiv gewendet aber die Vorzüge einer denkerischen Präzision hat, die eben nicht im Aufbau eines geschlossenen Gedankengebäudes gipfelt, sondern durch essayistische Annährungen geprägt ist: durch kluge Fragen an der Stelle altkluger Antworten, durch narrative Erkundungen und experimentelle Inszenierungen an der Stelle definitiver Aussagen. Als politischer Mensch war der Autor Max Frisch also weniger im Dialog mit einer bestimmten Tradition politischer Philosophie; er war vielmehr selber kreativ mit Entwürfen zu einer reflektierten politischen Positionierung des Intellektuellen, dem philosophische Dignität nicht abzusprechen ist. Es ist freilich eine Art des Schreibens, die den Moralisten näher steht als dem idealistischen Systemdenken oder der modernen analytischen Philosophie.

4 Der Regisseur Matthias von Gunten hat 2008 für den Titel seines Dokumentarfilms Max Frisch, Citoyen ${ }^{5}$ die treffende Kurzformel gefunden, um das politisch-ethische Anliegen auf den Punkt zu bringen: die Verantwortung des citoyen, der am Zeitgeschehen teilnimmt und sich öffentlich äußert, ohne in die Rolle des Oberlehrers zu schlüpfen. "Frisch war zu klug, um anderen irgendwelche Wahrheiten um die Ohren zu hauen“"6. 
Der Film dokumentiert auf anschauliche Weise mehrere Jahrzehnte politischer Zeitgenossenschaft und verzichtet auf lehrmeisterliche Gesten. Die von Frisch mit permanentem Selbstzweifel vorangetriebenen schriftstellerischen Projekte brachten ihn automatisch in Kontakt mit der politischen Aktualität, die er in sorgfältig redigierten Texten kommentierte. Deshalb sind sie auch heute noch mit großem Abstand zu der jeweiligen Tagesaktualität nachvollziehbar und lesenswert, auch wenn der von Frisch verkörperte Typus des Intellektuellen der Vergangenheit anzugehören scheint $^{7}$. Am Ende seines Lebensweges steht eine Haltung der Desillusionierung und der Skepsis. Dennoch bleibt Frisch bis zum Schluss der Aufklärer, der um die Dialektik der Aufklärung weiß und zur Kenntnis nimmt, dass viele Versprechen einer besseren und gerechteren Welt zu bitteren Enttäuschungen geführt haben. In seiner Rede bei den 8 . Solothurner Literaturtagen am 10. Mai 1986 hält er gerade im Wissen um die möglichen Perversionen einer zynisch und gewalttätig werdenden Rationalität am Projekt der Moderne fest. "Ohne einen Durchbruch zur sittlichen Vernunft, der allein aus Widerstand kommen kann, gibt es kein nächstes Jahrhundert, fürchte ich. Ein Aufruf zur Hoffnung ist heute ein Aufruf zum Widerstand ${ }^{\text {" }}$. Es ist der Widerstand gegen die Götzen eines marktliberalen Weltbildes, das alle Hoffnung in die Steigerung des Profits verlagert hat. Für Frisch ist dies der Tanz ums Goldene Kalb, die Unterordnung unter die behauptete Alternativlosigkeit des Ökonomischen, der Frisch konsequent den Primat des Politischen entgegensetzt. Die prinzipielle Verbundenheit mit einem Projekt der Moderne, die ihre Schattenseiten nur um den Preis der Selbsttäuschung verbergen kann, zieht sich wie ein roter Faden durch das Lebenswerk des Autors, der mit seinen öffentlichen Interventionen Bausteine einer politischen Ethik entwickelt hat, die gar nicht beansprucht, ein definitives System zu repräsentieren, die aber gerade in ihrem fragmentarischen Charakter um so interessanter ist für die Lernprozesse eines politischen Bewusstseins, das mit den Extremen des menschenverachtenden Totalitarismus und der an sich selbst zweifelnden Demokratie konfrontiert ist. Für Frisch steht fest, dass Demokratie mehr sein muss als ein "Urnenvolksspiel“". Auf die Frage, wie das Ethos der Demokratie mit Leben zu erfüllen sei, hat er im Laufe seines Lebens eine Reihe differenzierter Antworten gegeben, deren Konflikte und Widersprüche nicht verschwiegen werden sollen. Die Themenfelder und Argumentationsstrategien dieser politischen Ethik werden exemplarisch in den folgenden drei Bereichen untersucht: erstens am Beispiel der Bestimmung des Verhältnisses zwischen der Schweiz und Deutschland, zweitens mit einem Blick auf die großen Debatten im Zusammenhang mit Frischs Heimat-Diskurs über die Schweiz und drittens in den weltpolitischen Dimensionen der Orte, die der Autor auf der internationalen Bühne kennengelernt hat. Die drei Themenfelder werden abschließend zu der Frage zusammengeführt, ob und inwieweit die Literatur sich als Medium einer kosmopolitischen Ethik eignet.

\section{Nähe und Distanz zu Deutschland}

5 Frischs Verhältnis zu Deutschland hat verschiedene Phasen durchlaufen. Von einer rückhaltlosen Bewunderung in der Zeit vor dem Zweiten Weltkrieg über die Perspektive des Soldaten in der Schweizer Armee während des Krieges bis zur Wahrnehmung der Nazi-Verbrechen und einer intensiven Beschäftigung mit der Schuldfrage sowie einer neuen Annäherung an das Nachkriegsdeutschland mit Kontakten zu Autoren und Politikern, insbesondere während der Amtszeit von 
Bundeskanzler Helmut Schmidt. Die Wertschätzung, die Frisch in der Bundesrepublik Deutschland genoss, stand in einem merkwürdigen Kontrast zu den Verdächtigungen und Anfeindungen, denen er in der Schweiz ausgesetzt war. Doch kehren wir zunächst an den keineswegs unproblematischen Ausgangspunkt zurück: die unkritische Haltung des jungen Max Frisch vor 1939. Seine Freundschaft mit der emigrierten deutschen Jüdin Käte Rubensohn war ein Grund für einen Besuch in Berlin im Jahr 1935, also zu einem Zeitpunkt, als der Ungeist des nationalsozialistischen Regimes bereits unmissverständlich wahrnehmbar war.

Es spricht für Frisch, dass er dieses Kapitel seines Lebens nicht beschönigt hat. Auch in der neuen biografischen Gesamtdarstellung von Volker Weidermann ${ }^{10}$ wird schonungslos herausgearbeitet, dass die Reise nach Berlin, die ja eigentlich schon allein wegen Frischs Freundin ein Politikum hätte sein können, die naive Entdeckungsfahrt eines Unpolitischen war, der nur in geringem Umfang auf die Zustände in Deutschland reagierte. Im Auftrag der NZZ wollte er die Eindrücke in einem Tagebuch festhalten, "friedliche impressionen ... natürlich nichts scharfes ${ }^{\text {"11 }}$, wie er in einem Brief an Käte Rubensohn notierte. Die Beziehung zu seiner Freundin ging in die Brüche. Die Suche nach der Identität als Schreibender setzte sich fort, parallel zum Studium der Architektur und dem aktiven Militärdienst als Kanonier ab 1939. Diese Erfahrung wird Max Frisch ein Leben lang beschäftigen - nicht mit glorifizierenden Absichten, sondern mit dem nüchternen Blick auf die Anpassungsfähigkeit eines unpolitischen Menschen, der die Befehlsstrukturen seiner Armee zunächst nicht in Frage stellt und der die Idealisierung der Landesverteidigung unproblematisch findet. Erst als gegen Kriegsende das Ausmaß der Verbrechen in Deutschland und den besetzten Ländern unübersehbar ist, regt sich Frischs Gewissen. Seine Theaterstücke aus der zweiten Hälfte der 1940er Jahre sind Auseinandersetzungen mit der Schuldfrage und Versuche, die eigene Fassungslosigkeit zu verstehen. Der Autor fand eine Sprache, die es auch im kulturellen Vakuum der Nachkriegszeit in Deutschland ermöglichte, über das geschehene Unrecht ins Gespräch zu kommen.

7 Frischs Stücke verurteilen nicht. Sie sind Versuche, das Unfassbare zu verarbeiten und eine adäquate Form für diese Trauerarbeit zu erfinden. Leitmotivartig kehrt die Frage wieder: Was hätte ich getan, wenn ich einer vergleichbaren Situation gewesen wäre wie deutsche Befehlsempfänger, die nicht zu widersprechen wagten? Frisch beteiligte sich an Initiativen zu einer Normalisierung der Beziehungen zwischen der Schweiz und Deutschland und nutzte solche Gelegenheiten, um sein Kulturverständnis darzulegen. Bei einer Veranstaltung in Zürich im Jahr 1949 wirbt er dafür, dass Schweizer gar nicht anders können als sich mit Deutschland zu befassen. Denn die "räumliche Nachbarschaft" und die "Geschwisterschaft in der Sprache" sind Fakten, die nicht geleugnet werden können ${ }^{12}$. "Wenn Menschen, die die gleichen Worte sprechen wie ich und eine gleiche Musik lieben wie ich, nicht davor sicher sind, Unmenschen zu werden, woher beziehe ich fortan meine Zuversicht, dass ich davor sicher bin?" ${ }^{{ }^{13}}$ Frisch gelangt anlässlich dieser Überlegungen zu einer Klärung des Verhältnisses von Kultur und Politik, zu der er als Unpolitischer zehn Jahre vorher noch nicht fähig war. Er kritisiert nun die Aufspaltung des Lebens in die vermeintlich niederen Sphären der Politik und die Erhabenheit des geistigen Menschen. Eine solche Deutung von Kultur, die der Wirklichkeit entrückt ist, sei nichts anderes als Götzendienst, eine "sittliche Schizophrenie " ${ }^{14}$, die zur Katastrophe führen musste, weil es kein kritisches Korrektiv gab, um dem Wirklichkeitsverlust einer rein ästhetischen Kultur entgegenzuwirken. 
Trotz der Selbstverpflichtung des Schreibenden auf die Arbeit an der Form lehnt Frisch jegliche Art von Ästhetizismus ab, da diese niemals als Alibi für menschliches Versagen und politische Verantwortungslosigkeit überzeugen kann.

8 Als Frisch 1958 in Darmstadt mit dem Georg-Büchner-Preis ausgezeichnet wird, nutzt er die Gelegenheit, um die Rolle seiner Heimatstadt Zürich als Ort der Emigranten zu würdigen, wo auch Büchner als Autor des politischen Engagements Zuflucht fand. Frisch fühlt sich ihm verbunden im Geist des "Emigrantischen“, einer Haltung der Freiheit, die das "unausgesprochene Gefühl der Unzugehörigkeit" ${ }^{\text {“15 }}$ zur Folge hat. Damit ist ein Thema angesprochen, das sich für die implizite politische Ethik des Schriftstellers als zentral erweisen sollte.

\section{Schweiz als Heimat}

Als Frisch im Ausland längst ein angesehener und mit vielen Auszeichnungen geehrter Schriftsteller war, erhielt er 1974 in seiner Heimatstadt Zürich im festlichen Rahmen des Schauspielhauses, einer wichtigen Stätte seiner Arbeit als Dramatiker, den Großen Schillerpreis. Mit der Dankesrede gelang ihm einer der prägnantesten Texte seines öffentlichen Wirkens: die rhetorisch bemerkenswerten Reflexionen über den Begriff der Heimat. "Eine Ehrung aus der Heimat (und so sehe ich den Anlass hier und bin bewegt) weckt vor allem die Frage, was eigentlich unter Heimat zu verstehen ist" ${ }^{\star 16}$. Es ist die Dekonstruktion eines kitschigen, ideologisch vereinnahmten Heimatdiskurses, der in erster Linie mit ausgrenzender Absicht verwendet wird und dessen Verlogenheit Frisch nicht akzeptieren will. Seine eigene Sondierung des schwierigen Themas erfolgt mit viel Fingerspitzengefühl und mit Sinn für Humor. Es ist eine pointierte Skizze der politisch-ethischen Grundproblematik der Zugehörigkeit, dem ersten Gut, das ein Gemeinwesen zu verteilen hat. Über diese Frage wird meist deshalb nicht nachgedacht, weil sie administrativ vorentschieden zu sein scheint. Wir sind Bürger eines bestimmten Staates und mit entsprechenden Dokumenten ausgestattet, auf die wir Anspruch haben, weil wir in eine ganz bestimmte Situation hineingeboren wurden. In der Regel ist es aber nicht mehr als der Zufall der Geburt, der uns mit Anspruchsrechten ausstattet, die all jenen verweigert werden können, die unter anderen Bedingungen zu Bewohnern des jeweiligen Landes werden - zum Teil mit einem prekären Aufenthaltsstatus ${ }^{17}$.

Max Frisch versucht sich über Lexikondefinitionen dem Thema zu nähern und stößt sehr rasch auf die emotionale Komponente des Problems. Gefühle der Geborgenheit sind diffus und sind mit einer Vielzahl von Phänomenen in Verbindung zu bringen: mit der Vertrautheit eines Wohnviertels, den Strukturen einer Landschaft, den Klängen einer Sprache in Mundart und schriftsprachlicher Variante. All diese Elemente ergeben eine dichte Verknüpfung von Erfahrungen, die sehr widersprüchlich sein können. Das Spektrum geht von der Sehnsucht nach Beheimatung bis zur Entfremdung und zum Leiden an einer Heimat, die man nicht aufrichtig lieben kann und von der man sich auch nicht geliebt fühlt. Frisch fragt, wer sich die Definitionsmacht in solchen Angelegenheiten anmaßen darf. Wer "hätte denn das schlichte Recht, uns die Heimatliebe abzusprechen?" ${ }^{\prime 18}$ Der Schreibtisch des Schriftstellers kann an vielen Orten der Welt seinen Platz finden. Wichtig ist nur, dass die Umgebung inspirierend ist und dass Freunde in der Nähe sind, die jenen emotionalen Halt geben, ohne den kein Mensch glücklich wird. Doch bei aller Weltläufigkeit ist es unmöglich, seiner Herkunft 
ganz $\mathrm{zu}$ entrinnen und den Einfluss gemeinsamer Sprache und gemeinsamer Geschichte zu ignorieren. "Trotzdem zögere ich zu sagen: MEINE HEIMAT IST DIE SCHWEIZ. Andere sagen SCHWEIZ und meinen etwas anderes" ${ }^{\text {"19 }}$. Weil Frisch sich so vehement dagegen wehrt, Heimat mit Behaglichkeit zu verwechseln, kann er für sich beanspruchen, auch mit negativen Gefühlen - "in Zorn und Scham“"20 - als Schweizer mit seiner Heimat verbunden zu sein und Missstände zu beklagen.

11 Frisch hatte in der Schweiz weitaus mehr als im Ausland mit dem Image zu kämpfen, er wolle sich als ewiger Querulant profilieren und habe keinen Respekt vor den Leistungen seines Heimatlandes. Die Zürcher Rede aus Anlass der Verleihung des Schillerpreises belegt eindrücklich das Leiden des Autors an einer politischen und kulturellen Herkunft, von der er sich nicht lossagen möchte, deren Bestimmung er aber auch nicht einem konservativen kulturpolitischen Establishment überlassen will. In Frischs Wahrnehmung leidet der offizielle Identitätsdiskurs der Schweiz darunter, durch die Fixierung auf eine mythische Vergangenheit gelähmt zu sein und keine utopischen Potenziale zuzulassen. Ein Land ohne Utopien hat aber keine Kraft, die Zukunft zu gestalten und sich ehrgeizige Ziele zu setzen, wenn die kurzfristigen Notwendigkeiten sich immer auf Traditionspflege und Besitzbestandswahrung beschränken. Utopisches Denken ist ein heikles Thema der Moderne, weil nach dem Scheitern der utopischen Ideologien, die zu totalitären Regimen führten, Ernüchterung eingekehrt ist. Die Träume von einem besseren Menschen und einer gerechteren Gesellschaft endeten oft in Unfreiheit, Gewalt und Terror. 1960 beklagt Frisch, dass in der Schweiz das Wort ,Utopie" vorwiegend negativ verwendet werde, als sei schon Gefahr im Verzug, wenn Menschen nur anfingen, radikale Wünsche zu formulieren und sich Alternativen zur etablierten Ordnung auszudenken ${ }^{21}$.

Wie sehr sich Frisch eine solche Radikalität des freien Denkens wünschte, hatte er einige Jahre zuvor durch die Beteiligung an einer konkreten Utopie demonstriert. Anlass waren die langfristigen Vorbereitungen für eine Landesausstellung, eine nationale Leistungsschau, die schließlich 1964 in Lausanne durchgeführt wurde und an die erfolgreiche Ausstellung von 1939 in Zürich anknüpfen sollte. Das Zürcher Ereignis hatte ganz unter dem Eindruck der Landesverteidigung gestanden und war im kollektiven Bewusstsein ein wichtiger Teil der mentalen Einstimmung auf die Rolle der Schweiz im Schatten des drohenden Weltkriegs. Unter den veränderten Vorzeichen des Kalten Krieges sollte 25 Jahre später eine neue Beschwörung Schweizer Tugenden und Traditionen den Zusammenhalt in einem prosperierenden Land verstärken und stolz die Leistungen der Nachkriegszeit in Industrie, Technik und Kultur dokumentieren. Angesichts der erhofften Impulse wirkte Frischs Zwischenruf irritierend. Er war nämlich mit einigen Freunden entschieden der Auffassung, dass die Schweiz gut und gerne auf das überflüssige nationale Spektakel einer Landesausstellung verzichten könne und das dafür vorgesehene Geld besser in kreativere und zukunftsträchtigere Projekte zu investieren wäre. Dazu machte er 1955 in der Broschüre achtung: die Schweiz ${ }^{22}$ einen ungewöhnlich präzisen Vorschlag. Er fordert den Bau einer Musterstadt, in der neueste städtebauliche und ästhetische Prinzipien zu erproben seien und ein radikaler Bruch mit der Schweizer Städteplanung zu vollziehen sei. Das Mittelland sei ja nichts anderes als eine zersiedelte Landschaft, eine Verstädterung ohne Konzept und ohne anspruchsvolle Ideale. Museal gepflegte Stadtkerne wurden umringt von langweiligen Schlafstädten und verdichteten sich nach und nach zu einer urbanisierten Infrastruktur, die das Gebiet vom Genfer See bis zum Bodensee prägt. 
Peter Bichsel erzählt in Matthias von Guntens Film, dass er $1955 \mathrm{zu}$ den begeisterten Lesern der Broschüre gehörte, die die Anmeldekarte im Anhang ausfüllten, um ihre Bereitschaft zur Mitarbeit an der Verwirklichung der Utopie zu bekunden. "Im Ernst: gründen wir eine Stadt. Genauer gesagt: versuchen wir es. Denn darin besteht das Wesentliche: dass es ein Experiment ist. Es soll uns zeigen, ob wir noch eine lebendige Idee haben, eine Idee, die eine Wirklichkeit zu zeugen vermag, eine schöpferische

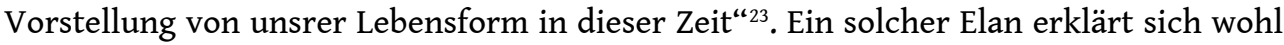
nicht nur aus dem Erfahrungshintergrund des gelernten Architekten, auch wenn diese professionelle Dimension eine Rolle gespielt haben mag, wie der Verweis auf das Vorbild Le Corbusiers zeigt. Frisch und seine Mitstreiter wollen die wohlhabende Schweiz aus der Lethargie reißen und zum Träumen und Handeln ermuntern: zum Abschied von dem dörflichen Kolorit der Gründungsmythen des Landes und zum Experimentieren mit den neuen Bedingungen von Verkehr, Kommunikation und Wohnformen. "Es geht um die Manifestation einer schweizerischen Lebensform, um die Frage des Stils “" ${ }^{{ }_{24}}$. Aus heutiger Sicht stellen viele Enthusiasten von damals mit Selbstironie fest, es sei besser, dass das Projekt von 1955 nicht verwirklicht werden konnte. In der kalten und rechteckigen Betonstadt im Stil von Le Corbusier hätten sich möglicherweise nur wenige Menschen wohlgefühlt. Doch allein die Ausformulierung einer solchen Idee belegt Frischs politisches Temperament, das zur Aktion drängt, auch wenn der Schriftsteller Max Frisch sich von aktionistischen Konzepten einer engagierten Literatur distanziert. Er artikuliert sich in der Rolle des Intellektuellen und des Citoyen, der seine Prominenz nutzen kann, um Zustände zu kritisieren, kreative Tendenzen zu verstärken und Debatten zu stimulieren. Dass er sich damit auch Missverständnisse und Feindschaften einhandelte, war ihm klar, zumal das Thema der Planung einer Musterstadt einen schlechten Beigeschmack hatte in einer Zeit, in der jeder Ruf nach Planung leicht als Sympathie mit sozialistischer Planwirtschaft diskreditiert werden konnte.

Frischs Interventionen als Intellektueller lassen keinen Zweifel daran, dass seine tiefste Motivation nicht die Eitelkeit des prominenten Autors ist, sondern die politischethische Überzeugung von der Demokratie als Lebensform. Gerade deshalb leidet er auch so stark unter der Politikverdrossenheit seiner Mitbürger, die nur schwer für neue Ideen zu begeistern sind. Demokratie lebt von der Kontroverse, vom Ausprobieren ungewöhnlicher Programme, von wechselnden Mehrheiten und vom Respekt vor abweichenden Meinungen. All das ist in einer Konkordanzdemokratie nur schwer zu bewerkstelligen. Obwohl der Schweizer Bürger über mehr Mitwirkungsrechte verfügt als der Bürger einer konventionellen parlamentarischen Demokratie, schöpft er diese Möglichkeiten nur selten aus, weil der Konsens höher geschätzt wird als die offenlegung der Differenzen. So gehörte Frisch zu jenen Mahnern, die nicht müde wurden davor zu warnen, dass hinter der Fassade legitimierter Institutionen die politische Macht sich in den Händen von wenigen konzentrierte, die oft neben dem politischen Einfluss auch über die ökonomischen Entscheidungsstrukturen verfügten. "Kann unsere Demokratie-Utopie, ausgesetzt der menschlichen Natur, also der Canaille, die der Mensch in der Mehrheit ist, zu etwas anderem führen als zu der real existierenden Demokratie der Lobbies, getarnt durch Folklore? - oder mit anderen Worten: Wieviel wirkliche Demokratie (Volk als Souverän) ist im real existierenden Kapitalismus überhaupt möglich?“"25 So fragte Frisch 1987 anlässlich eines Podiumsgesprächs. Es sind mehr als nur radikale Voten eines zornigen alten Mannes. Dahinter stehen die Kontinuität von Jahrzehnten kritischer Beobachtung der Erosion 
demokratischer Ideale und die Solidarisierung mit all jenen, die sich für demokratische Teilhaberechte einsetzen. Frischs Reaktionen auf die Zürcher Jugendrevolte und andere Interventionen im Jahr 1968 belegen das sehr deutlich ${ }^{26}$.

15 Es gibt einen Politikbereich, der wie kaum ein anderer als Testfall dafür angesehen werden kann, wie weit die Integrationskraft einer Demokratie geht und wie ernst es mit der Idee ist, aus allen Menschen, die durch ihre Arbeit zum Wohlstand eines Landes beitragen, aktive Bürgerinnen und Bürger zu machen. Es ist der Bereich der Integration von Fremden. Max Frisch hat das Verdienst, dieses Thema von Anfang an im Blick gehabt zu haben und eine politisch-ethische Konkretisierung dessen zu entwickeln, was er später im Nachdenken über den Begriff der Heimat verdichtete. Der Text Überfremdung $I^{27}$ dokumentiert eine der ersten öffentlichen Interventionen des Autors nach einem mehrjährigen Aufenthalt in Rom. Vielleicht fühlte er sich gerade durch diese italienischen Jahre gedrängt, sich zur schlechten Behandlung der italienischen Gastarbeiter in der Schweiz zu äußern, obwohl er sich fest vorgenommen hatte, mit scharfer Kritik an der Schweiz etwas zurückhaltender zu sein ${ }^{28}$. Der knapp drei Seiten umfassende Text ist eine Miniatur von großer Ausdruckskraft. Der zweite Teil des Eröffnungssatzes ist eine der am häufigsten zitierten Wendungen in der sozialwissenschaftlichen und philosophisch-ethischen Literatur zu Fragen der Migration: "Ein kleines Herrenvolk sieht sich in Gefahr: man hat Arbeitskräfte gerufen, und es kommen Menschen ${ }^{\text {“29. }}$. Diese Formulierung ist ein Paukenschlag: die lapidare Beschreibung der Arbeitsmigration als einer aus wirtschaftlichen Eigeninteressen initiierten Zuwanderung unter Ausblendung der Tatsache, dass Menschen mit ihren legitimen Bedürfnissen kommen - über die für die Konjunktur wichtige Arbeitsleistung hinaus. Dieser genialen Kurzformel wird die ebenso lapidare und provokative Bemerkung vorangestellt, dass "ein kleines Herrenvolk" auf die eigentlich von ihm gewollte Zuwanderung nervös reagiert. Sprachlich wird der Zusammenhang mit der Nazizeit hergestellt, als die Schweiz sich durch das feindselige "Herrenvolk" nördlich der Grenze in Gefahr sah.

Die ausländischen Arbeitskräfte der frühen 60er Jahre, in Deutschland Gastarbeiter, in der Schweiz Fremdarbeiter genannt, kamen überwiegend aus Italien, sprachen also eine Sprache, die immerhin eine der Landessprachen der Schweiz ist. Das genügte aber nicht, um das Gefühl von Fremdheit und Bedrohung zu reduzieren. Frisch charakterisiert in prägnanten Sätzen die wirtschaftlichen, kulturellen, vor allem aber menschlichen Facetten des Phänomens. Die wirtschaftlichen Interessen bei der Anwerbung ausländischer Arbeitskräfte schienen so sehr im Vordergrund gestanden zu haben, dass die Verantwortlichen in Wirtschaft und Politik sich überhaupt keine Gedanken darüber gemacht hatten, wie ein menschliches Miteinander mit den Fremden hätte aussehen können, sobald diese im Alltag der Schweizer Gesellschaft unübersehbar wurden. Das Unbehagen der Einheimischen wird durchaus als Widerspruch zum Mythos Schweiz wahrgenommen, da dieses Land sich ja bewusst als tolerant, weltoffen und antirassistisch verstanden wissen wollte. Frisch spricht einer "Hysterie der Hilflosigkeit" und von der Notwendigkeit, "den Begriff der Schweiz in die

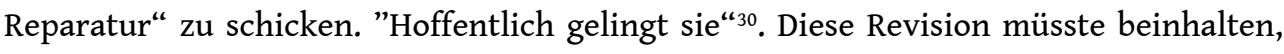
ein anderes Verhältnis zu fremden Sprachen und Lebensformen zu entwickeln. Wie sehr Max Frisch mit seiner Diagnose den Nerv seines Landes getroffen hat, lässt sich daran ablesen, wie sehr bis heute rechtspopulistische Kreise in der Schweiz mit einer diffusen Überfremdungsangst in der Bevölkerung Stimmung machen und damit Abstimmungserfolge erzielen. Offensichtlich ist es mit Frischs hellsichtiger Situierung 
des Problems ${ }^{31}$ nicht gelungen, einen Mentalitätswandel einzuleiten. Die von ihm so beklagte Abwehrhaltung, die auf die Bewahrung einer Schweizer Eigenart gerichtet ist und sich jeder kreativen und angstfreien Problemlösung verweigert, ist längst nicht verschwunden.

Die grobe Skizze von Frischs politisch-ethischen Stellungnahmen zum Schweizer Selbstverständnis wäre unvollständig, wenn nicht noch eine der symbolisch wichtigsten Institutionen des Landes zur Sprache käme: die Armee. Frisch äußert sich dazu in mehreren Lebensphasen: zunächst durchaus affirmativ aus der Perspektive des aktiven Militärdienstes ab 1939, dem schon die 1931 abgeschlossene Rekrutenschule vorausgegangen war. Die Blätter aus dem Brotsack ${ }^{32}$ erschienen als Tagebuch des Soldaten erstmals 1940. 1974 kam es zu einer kritischen Wiederaufnahme des Themas im Dienstbüchlein ${ }^{33}$ und schließlich 1989 zu einer der letzten großen politischen Stellungnahmen des Autors in dem Text Schweiz ohne Armee $?^{34}$, der unter dem Titel Jonas und sein Veteran für die Bühne bearbeitet und deutsch in Zürich und französisch in Lausanne uraufgeführt wurde. Hintergrund dieser mit lebhaften gesellschaftlichen Debatten verbundenen Inszenierungen war die Volksinitiative Für eine Schweiz ohne Armee und für eine umfassende Friedenspolitik, die von der Gruppe für eine Schweiz ohne Armee (GSoA) 1986 auf den Weg gebracht worden war und im November 1989 zur Abstimmung kam. Die Initiative scheiterte zwar, konnte aber mit 35,6\% der Stimmen für die Abschaffung der Armee einen Achtungserfolg erziehen, der auch bemerkenswert ist, weil die Stimmbeteiligung mit $68,6 \%$ überdurchschnittlich hoch war. Dass Frisch, der eigentlich nicht mehr publizieren wollte, sich mit einem eigens für diesen Anlass verfassten Text einmischte, unterstreicht die Bedeutung, die er dem Thema beimaß, das 1989, also 50 Jahre nach der Mobilmachung, auch ohne die Volksinitiative die politische Agenda der Schweiz bestimmt hätte.

Der Text präsentiert sich nicht als friedenspolitischer Traktat, sondern als lockerer Dialog zwischen Jonas und seinem Großvater, der leicht mit dem Autor zu identifizieren ist. Dem Haupttext ist ein Glossar beigefügt, in dem zentrale Sachverhalte aus dem Schweizer Militärwesen erläutert werden. Das Palaver enthält längere Passagen aus dem Dienstbüchlein von 1974, das der Großvater am Ende des Gesprächs demonstrativ ins Kaminfeuer wirft. Die Pointe des Palavers ist das Insistieren auf der Unmöglichkeit einer konsequenten Friedenspolitik, da diese auf dem radikalen Umbau der Gesellschaft beruhen müsste. Frisch hat diesen Gedanken wenige Tage vor der Abstimmung nochmals unterstrichen. "Friedensfähig wäre eine Gesellschaft, die ohne Feindbilder auskommt. Wer kann sich das innenpolitisch leisten! Gesellschaften mit Gewalt-Struktur mögen sich den Nicht-Krieg wünschen; der Friede widerspräche ihrem Wesen. Was ihnen reicht: der Nicht-Krieg ““35. Frisch rechnete nicht ernsthaft mit einem Abstimmungserfolg der Volksinitiative. Für ihn war es schon eine Genugtuung $\mathrm{zu}$ sehen, dass es der Initiative gelungen war, eine breite Debatte $\mathrm{zu}$ lancieren, die Verantwortlichen in der Armee zu verunsichern und das Bewusstsein für eine demokratische Gesellschaft zu stärken. Ab diesem Moment ist es nämlich nicht mehr möglich, sich mit dem Argument zu begnügen, dass die Armee ja immerhin 50000 Arbeitsplätze garantiere und schon allein deshalb gar nicht abgeschafft werden könne. Dagegen stellt Frisch die revolutionäre Hoffnung auf die prinzipielle Wünschbarkeit und Machbarkeit eines Umbaus der Gesellschaft. Auch in dieser Frage äußert er sich nicht mit philosophischen Prätentionen, sondern mit dem Verantwortungsbewusstsein des Citoyen, der die Macht des Wortes im Einsatz für seine moralischen Überzeugungen zu nutzen versteht. Frischs Haltung beruht nicht auf 
einem radikalen Pazifismus, sondern auf einer Analyse politischer Mythen, unter denen die Beschwörung der Verteidigungsbereitschaft und der wehrhaften Demokratie in der Schweiz an erster Stelle steht. Dass eine solche Geschichtsschreibung zu korrigieren ist, war für Frisch nicht zuletzt deshalb wichtig, weil er als junger Soldat noch vorbehaltlos an die Mythen der Schweiz geglaubt hatte.

\section{Weltbürgertum}

Das Porträt der politischen Dimension in Max Frischs intellektueller Präsenz wäre unvollständig ohne die Erwähnung seiner internationalen Ausstrahlung. Der Autor, der sich ein Leben lang an seinem Heimatland abgearbeitet hat, muss an diesem Land, ganz besonders auch an der Stadt Zürich, sehr gehangen haben. Sonst wäre die Intensität so mancher Kritik nicht zu begreifen. Den kritischen Blick auf die Schweiz konnte sich Frisch auch deshalb leisten, weil er das Land immer wieder aus größerem Abstand betrachtete: durch die längeren Aufenthalte in den USA und in Italien und durch die zahlreichen Reisen, die ihn mit vielen geopolitischen Brennpunkten des Kalten Krieges in Berührung brachten. Am Ende seines Lebens hat er zwar das Ende dieser weltweiten Machtkonstellation erleben können. Seine aktiven Jahre waren aber vor allem durch den Kalten Krieg und die damit verbundenen Mentalitäten und Dispute geprägt. In allen politischen Kontakten dieser Zeit war er als Kulturschaffender stets auf seine Unabhängigkeit bedacht und ließ sich vor keinen ideologischen Karren spannen. Seine vorzeitige Abreise vom 1948 in Breslau veranstalteten Weltkongress der Intellektuellen für den Frieden gibt davon ein beredtes Zeugnis ${ }^{36}$. Der doktrinäre Ton von Parteifunktionären und der stromlinienförmige Opportunismus eigener westlicher Intellektueller waren ihm gleichermaßen ein Graus.

20 Auch die Entdeckung der amerikanischen Wirklichkeit blieb von aufschlussreichen Nuancierungen geprägt. Die Arroganz der US-amerikanischen Supermacht war Frisch ebenso suspekt wie die Arroganz der Europäer im Umgang mit dem als kulturell unterlegen empfundenen Amerika. Eine Frucht seines USA-Aufenthalts in den Jahren 1951 und 1952 ist ein Text, in dem er die herablassenden Reaktionen auf Amerika kritisiert. Aus heutiger Sicht liest sich dieser Aufsatz wie eine visionäre Vorwegnahme dessen, was wir unter dem Stichwort Globalisierung diskutieren und was schon Frisch als die Geburt des globalen Menschen thematisierte. "Die Erde fängt an, rund zu werden auch im Erlebnis der Menschen, nicht bloß in der Kenntnis“" ${ }^{\text {“37 }}$. Auf der theoretischen und praktischen Ebene bedeutet dies einen konsequenten Abschied von einem hochnäsigen Eurozentrismus, der auch für die westliche Philosophie so selbstverständlich war und erst langsam einem Gespür für interkulturelle Vernetzungen Platz macht. "Der Typus des globalen Menschen aber wird erst geboren, und zwar, wie mich dünkt, vor allem in Amerika, das, wie gesagt, nicht ein Land ist, sondern ein Kontinent, nicht von einem Volk bewohnt, sondern von einer Völkerwanderung. Und dass dieser Typus des globalen Menschen sich weigern wird, Europa als die geistige Weltmitte zu betrachten, ist kein Grund für den Europäer, arrogant zu werden; kein Grund, den Mut zu verlieren. Europa ist wichtig, aber es ist nicht die Menschheit, nicht, die Kultur"“38.

21 Die heilsame Relativierung des Bewusstseins, in Europa oder gar in der Schweiz den Mittelpunkt der Welt zu repräsentieren, schafft Freiräume für neue Erfahrungen, neue Quellen der literarischen Inspiration und neue Orte der intellektuellen Beteiligung an 
den Strukturen einer im Entstehen begriffenen Weltgesellschaft. Die prinzipiellen Sympathien für Amerika haben Frisch nie daran gehindert, seine Kritik an der Außenpolitik der USA im Kontext des Vietnamkriegs deutlich zu äußern. Sein weltbürgerliches Engagement war ihm erst recht möglich geworden, nachdem er den Mythos von der Schweizer Neutralität entzaubert hatte. Frischs Romane, Tagebücher und Theaterstücke geben nicht weniger als seine Reden Aufschluss über ein Werk, das überall Resonanz finden kann, weil es die Lebenserfahrungen und Phantasien eines Weltbürgers spiegelt, der prinzipiell für alle Anregungen offen ist.

\section{Literatur als Praxis einer kosmopolitischen Ethik}

Max Frischs intellektuelle Biografie liest sich wie eine Serie von Experimenten mit räumlicher Nähe und Distanz. Das Spannungsverhältnis von Eigenem und Fremden, von prägender Herkunft und offener Zukunft, von Beheimatung und nomadischer Existenz, von Identifikation und Verfremdung spiegelt sich selbst im Symbolwert der beiden Schweizer Domizile: dem urbanen Zürich und dem abgelegenen Tessiner Tal, die beide Ausgangspunkte waren für längere Reisen. Der Architekt Frisch hatte nicht das geringste Interesse am Bau eines eigenen Hauses, sondern lebte lieber in Provisorien und in der offenheit für neue Lebenserfahrungen. In dieser Hinsicht steht er exemplarisch für den Typus des Kosmopoliten, der den Wert der Freundschaft zu schätzen weiß, der sich aber auch in einer Welt von Fremden orientieren kann und Fremdheit als Bereicherung seiner Identität empfindet ${ }^{39}$. Insofern kann es nicht überraschen, dass er zu einem Autor von weltliterarischem Rang wurde, wie es Mario Vargas Llosa anlässlich von Stiller gewürdigt hat ${ }^{40}$, und zwar gerade in Auseinandersetzung mit der komplizierten Schweizer Heimat. "Wohlhabend, gebildet und frei zu sein, ist anscheinend von tödlicher Langeweile. Der Preis, den man für den Genuss derartiger Privilegien zahlt, ist die Monotonie der Existenz, ist ein endemischer Konformismus, der Verlust der Phantasie, das Verschwinden des Abenteuers und eine Formalisierung der Emotionen und Gefühle, welche die Beziehungen zwischen den Menschen auf rituelle, substanzlose Gesten und Worte reduziert" ${ }^{41}$. Dennoch belegt, wie schon Vargas Llosa unterstreicht, die bloße Existenz des von Widerspruchsgeist geprägten Werks Frischs die literarische Produktivität einer solchen Umgebung.

Max Frisch hat in seinem Werk darauf geachtet, die Gattungsunterschiede zwischen Literatur und Philosophie ${ }^{42}$, Literatur und intellektuellem Engagement, Kunst und Politik zu respektieren und gleichzeitig Übergänge zwischen den Bereichen $\mathrm{zu}$ ermöglichen. Er führte die Perspektiven bereits 1958 zusammen in einem Begriff von Öffentlichkeit ${ }^{43}$, der die Möglichkeiten der Kommunikation mit einem Publikum auslotet. Eine solche Resonanz ist mehr als je zuvor weltweit möglich und schafft neue Erfahrungsräume einer Solidarität unter Fremden. Wer als Schriftsteller Erfolg hat, wird vom Publikum in die Rolle dessen gedrängt, der auch zu Fragen außerhalb der Literatur kluge Meinungen formulieren soll. Frisch hat diese Rolle akzeptiert, weil sie mit seinem moralischen und politischen Selbstverständnis als Citoyen gut $\mathrm{zu}$ vereinbaren war. Im Rückblick bestätigt sich seine selbstkritische Einschätzung, kein Philosoph zu sein. Doch gerade diese kluge Wahrnehmung der eigenen Talente hat es ihm ermöglicht, zu einer politisch-ethischen Stimme zu werden, die auch noch heutige ethische Debatten anregen kann. Seine späten friedenspolitischen Interventionen geringschätzig unter der Narrenfreiheit eines alten und kranken Mannes zu verbuchen, 
wäre eine Verkennung der Kontinuität eines Schreibens, das den eigenen moralischen Standards treu geblieben ist.

\section{NOTES}

1. Max Frisch, Entwürfe zu einem dritten Tagebuch (Herausgegeben und mit einem Nachwort von Peter von Matt), Frankfurt, Suhrkamp, 2010, S. 176.

2. Ebd., S. 177.

3. Vgl. beispielsweise Will Kymlicka, Contemporary Political Philosophy. An Introduction, Oxford / New York, Clarendon Press, 1990; Jonathan Wolff, An Introduction to Political Philosophy, Oxford / New York, Oxford University Press, 1996.

4. Entwürfe zu einem dritten Tagebuch, a.a.O., S. 177.

5. Als DVD in der Filmedition Suhrkamp, Frankfurt, Suhrkamp, 2009.

6. Matthias von Gunten im Gespräch mit Dietmar Kammerer, abgedruckt im Booklet zur DVD, S. 7.

7. Das liegt schon allein an der Veränderung der Medienlandschaft mit ihrer Vervielfachung der Kommunikationsformen und einer Beschleunigung der Mitteilungen, die für ausgefeilte Reden und inszenierte öffentliche Diskussionsanlässe immer weniger Zeit lässt.

8. Max Frisch, "Am Ende der Aufklärung steht das Goldene Kalb“, in: Max Frisch, Schweiz als Heimat? Versuche über 50 Jahre (Herausgegeben und mit einem Nachwort versehen von Walter Obschlager), Frankfurt, Suhrkamp, 1990, S. 461-469, hier 468.

9. Max Frisch, "Demokratie - ein Traum?", in: Schweiz als Heimat?, a.a.O., S. 489-492, hier 489.

10. Volker Weidermann, Max Frisch. Sein Leben, seine Bücher, Köln, Kiepenheuer \& Witsch, 2010.

11. Zit. nach Weidermann, ebd., S. 56.

12. Max Frisch, "Kultur als Alibi“, in: Max Frisch, Öffentlichkeit als Partner, Frankfurt, Suhrkamp, 1967, S. 15-24, hier 15.

13. Ebd., S. 20.

14. Ebd., S. 21.

15. Max Frisch, "Emigranten“, in: Schweiz als Heimat?, a.a.O., S. 181-194, hier 191.

16. Max Frisch, "Die Schweiz als Heimat?", in: Schweiz als Heimat?, a.a.O., S. 365-373, hier 365.

17. Trotz der grundlegenden Bedeutung dieser Fragen ist die Analyse von Mitgliedschaft und Zugehörigkeit in der politischen Philosophie bis vor kurzem marginal geblieben. Sie hat erst im Rahmen der neueren Migrationsforschung auch in Gerechtigkeitstheorien stärkere Beachtung gefunden. Vgl. Michael Walzer, Spheres of Justice. A Defense of Pluralism and Equality, Oxford, Blackwell, 1983, S. 31-63; Seyla Benhabib, Kulturelle Vielfalt und demokratische Gleichheit. Politische Partizipation im Zeitalter der Globalisierung, Frankfurt, Fischer Taschenbuch Verlag, 1999.

18. Ebd., S. 367.

19. Ebd., S. 370.

20. Ebd., S. 373.

21. Max Frisch, "Die Schweiz ist ein Land ohne Utopie", in: Schweiz als Heimat?, a.a.O., S. 195f.

22. Max Frisch, "achtung: die Schweiz. Ein Gespräch über unsere Lage und ein Vorschlag zur Tat", in: Schweiz als Heimat?, a.a.O., S. 133-179.

23. Ebd., S. 151.

24. Ebd., S. 162. 
25. Max Frisch, "Demokratie - ein Traum?“, in: Schweiz als Heimat?, a.a.O., S. 489-492, hier 490.

26. Max Frisch, "Demokratie ohne Opposition?“, in: Schweiz als Heimat?, a.a.O., S. 250-259.

27. In: Schweiz als Heimat?, a.a.O., S. 219-221.

28. "Vorsatz", in: Schweiz als Heimat?, a.a.O., S. 222.

29. "Überfremdung I“, in: Schweiz als Heimat?, a.a.O., S. 219.

30. Ebd., S. 221.

31. Vgl. auch die umfangreichere Entfaltung des kurzen Textes in "Überfremdung II", in: Schweiz als Heimat?, S. 223-244. Dieser Text geht auf einen Vortrag bei der Jahreskonferenz der kantonalen Fremdenpolizeichefs 1966 zurück. Allein die Tatsache einer solchen Einladung spricht dafür, dass es bei dieser Behörde durchaus ein Problembewusstsein gab, das als hoffnungsvolles Zeichen zu werten ist.

32. In: Schweiz als Heimat?, a.a.O., S. 9-69.

33. In: Schweiz als Heimat?, a.a.O., S. 377-455.

34. In: Schweiz als Heimat?, a.a.O., S. 493-547. Erstausgabe: Schweiz ohne Armee? Ein Palaver, Zürich, Limmat Verlag, 1989.

35. Max Frisch, "Der Friede widerspricht unserer Gesellschaft", in: Schweiz als Heimat?, a.a.O., S. 549-552, hier 550.

36. Vgl. Weidermann, Max Frisch, a.a.O., S. 133-135.

37. Max Frisch, "Unsere Arroganz gegenüber Amerika“, in: Öffentlichkeit als Partner, a.a.O., S. 25-34, hier 34.

38. Ebd., S. 35.

39. Vgl. Kwame Anthony Appiah, Cosmopolitanism. Ethics in a World of Strangers, London, Penguin, 2007.

40. Mario Vargas Llosa, "Ist es möglich, Schweizer zu sein?“, in: Luis Bolliger / Walter Obschlager / Julian Schütt (Hrsg), Jetzt: Max Frisch, Frankfurt, Suhrkamp, 2001, S. 104-109.

41. Ebd., S. 104.

42. Vgl. zur Bedeutung des Festhaltens an diesem Gattungsunterschied: Jürgen Habermas, Der philosophische Diskurs der Moderne, Frankfurt, Suhrkamp, 1985, S. 219-247; Jürgen Habermas, Nachmetaphysisches Denken, Frankfurt, Suhrkamp, 1988, S. 244.

43. Max Frisch, Öffentlichkeit als Partner, a.a.O., S. 56-67.

\section{RÉSUMÉS}

L'œuvre de Max Frisch a une dimension politique et éthique qui se manifeste dans ses nombreuses interventions publiques. Sans que ses contributions soient ancrées dans une tradition philosophique précise, elles partagent le projet des Lumières qui met l'émancipation et la liberté à la place de la sujétion. La conscience politique de l'auteur est éveillée par ses désillusions à la fin de la Seconde Guerre mondiale qui a démontré cruellement l'échec de l'humanité et de l'idéal des Lumières. Frisch propose une analyse nuancée des rapports avec l'Allemagne. Il provoque en Suisse des réactions par sa critique radicale des mythes fondateurs de son pays et de ses institutions, considérées comme intouchables. Ses réflexions sur la démocratie, la politique d'immigration et l'avenir de l'armée se lisent comme des témoignages de l'intégrité intellectuelle de quelqu'un qui n'a pas peur des conflits et qui préfère le débat d'idées au conformisme. Au-delà des frontières de la Suisse, l'attrait de Frisch s'explique par l'ouverture 
cosmopolite d'une littérature qui met l'accent sur l'originalité de l'expérience des autres et qui reste sceptique à l'égard de toute fixation précipitée d'une identité. Dans la tradition des Lumières dont Frisch connaît la face obscure, il tient aux idéaux utopiques de la modernité qu'on peut déjà expérimenter dans les espaces de liberté de la littérature, mais qui devraient également inspirer une gestion responsable de l'avenir politique.

The work of Max Frisch has a political and ethical dimension which is visible in numerous public contributions. Without being linked to one specific philosophical tradition, they all share the Enlightenment project of looking for emancipation and freedom instead of dependency. The political consciousness of the author was stimulated by the disillusion at the end of World War II that had demonstrated so terribly the failure of humanity and of the ideal of Enlightenment. Frisch looks for a nuanced evaluation of the relations with Germany and provokes his home country, Switzerland, by a radical critique of its founding myths and its untouchable institutions. His reflections on democracy, immigration policy and the future of the army can be read as a testimony to the intellectual integrity of someone who is not afraid of conflicts, and prefers controversial debate to prevalent conformism. Beyond the Swiss borders, Frisch's voice is attractive because of its cosmopolitan openness to a literature that is searching for subjectivity in the experience of otherness, and remains sceptical with regard to hasty fixations of identity. In the Enlightenment tradition, and aware of its darker side, Frisch sticks to the utopian ideals of modernity which can already be experienced in the free practice of literature, and which should also inspire the responsible shaping of the political future.

Max Frischs Werk beinhaltet eine politisch-ethische Dimension, die sich in zahlreichen öffentlichen Stellungnahmen manifestiert. Ohne diese Beiträge in einer bestimmten philosophischen Tradition zu verankern, ist ihnen doch der Anspruch der Aufklärung gemeinsam, Emanzipation und Freiheit an die Stelle von Abhängigkeit zu setzen. Das politische Bewusstsein des Autors wird stimuliert durch die Desillusionierung am Ende des Zweiten Weltkriegs, der die Möglichkeiten des Scheiterns von Humanität und Aufklärungsidealen drastisch vor Augen geführt hatte. Frisch sucht nach einer differenzierten Neubestimmung des Verhältnisses zu Deutschland und provoziert in seinem Heimatland Schweiz durch die radikale Kritik von dessen Gründungsmythen und als unantastbar geltenden Institutionen. Seine Gedanken über Demokratie, Einwanderungspolitik und die Zukunft des Militärs lesen sich als Zeugnisse der intellektuellen Unbestechlichkeit eines Menschen, der den Konflikt nicht scheut und eine lebhafte Debattenkultur lieber sähe als den vorherrschenden Konformismus. Über die Grenzen der Schweiz hinaus entwickelte die Stimme Frischs eine Anziehungskraft, die sich aus der weltbürgerlichen Offenheit einer Literatur erklärt, die das Eigene im Fremden sucht und voreilige Fixierungen von Identität skeptisch betrachtet. In der Tradition der Aufklärung, um deren Schattenseiten Frisch weiß, hält er an den utopischen Idealen der Moderne fest, die in den Freiräumen der Literatur zu erproben sind, aber auch eine verantwortliche Gestaltung der politischen Zukunft inspirieren sollten.

\section{AUTEUR}

\section{WALTER LESCH}

UCL Université catholique de Louvain Faculté de théologie \& Faculté de philosophie, arts et lettres 